\title{
Aerobic Exercise-Induced Changes in Cardiorespiratory Fitness in Breast Cancer Patients Receiving Chemotherapy: A Systematic Review and Meta-Analysis
}

\author{
Guilherme Maginador ${ }^{1}$, Manoel E. Lixandrão ${ }^{2}$, Henrique I. Bortolozo ${ }^{1}$, Felipe C. Vechin ${ }^{2}$, \\ Luís O. Sarian ${ }^{1}$, Sophie Derchain ${ }^{1}$, Guilherme D. Telles ${ }^{2}$, Eva Zopf ${ }^{3}$, Carlos Ugrinowitsch ${ }^{2} \mathbb{D}$ \\ and Miguel S. Conceição 1,2,4,*(D) \\ 1 Department of Obstetrics and Gynecology, Faculty of Medical Sciences, University of Campinas, Campinas, \\ São Paulo 13083-881, Brazil; guilherme.maginador@gmail.com (G.M.); hbortolozo@gmail.com (H.I.B.); \\ sarian@unicamp.br (L.O.S.); sophie.derchain@gmail.com (S.D.) \\ 2 School of Physical Education and Sport, University of São Paulo, São Paulo 05508-030, Brazil; \\ manelix.ef@gmail.com (M.E.L.); felipe.cassaro@yahoo.com.br (F.C.V.); guitelles11@hotmail.com (G.D.T.); \\ ugrinowitsch@gmail.com (C.U.) \\ 3 Department of Exercise Oncology, Mary MacKillop Institute for Health Research, Australian Catholic \\ University, Melbourne 3000, Australia; Eva.Zopf@acu.edu.au \\ 4 Faculty of Physical Education, University of Campinas, Campinas 13083-851, Brazil \\ * Correspondence: conceicao.miguel0106@gmail.com; Tel.: +55-11-3091-8733
}

Received: 21 June 2020; Accepted: 30 July 2020; Published: 11 August 2020

\begin{abstract}
While performing aerobic exercise during chemotherapy has been proven feasible and safe, the efficacy of aerobic training on cardiorespiratory fitness (CRF) in women with breast cancer undergoing chemotherapy has not yet been systematically assessed. Therefore, the objective of this work was to determine (a) the efficacy of aerobic training to improve CRF; (b) the role of aerobic training intensity (moderate or vigorous) on CRF response; (c) the effect of the aerobic training mode (continuous or interval) on changes in CRF in women with breast cancer (BC) receiving chemotherapy. A systematic review and meta-analysis were conducted as per PRISMA guidelines, and randomized controlled trials comparing usual care (UC) and aerobic training in women with BC undergoing chemotherapy were eligible. The results suggest that increases in CRF are favored by (a) aerobic training when compared to usual care; (b) vigorous-intensity aerobic exercise (64-90\% of maximal oxygen uptake, $\mathrm{VO}_{2 \mathrm{max}}$ ) when compared to moderate-intensity aerobic exercise (46-63\% of $\mathrm{VO}_{2 \max }$ ); and (c) both continuous and interval aerobic training are effective at increasing the $\mathrm{VO}_{2 \mathrm{max}}$. Aerobic training improves CRF in women with BC undergoing chemotherapy. Notably, training intensity significantly impacts the $\mathrm{VO}_{2 \max }$ response. Where appropriate, vigorous intensity aerobic training should be considered for women with $\mathrm{BC}$ receiving chemotherapy.
\end{abstract}

Keywords: aerobic fitness; breast cancer; exercise rehabilitation; $\mathrm{VO}_{2 \max }$

\section{Introduction}

According to the World Health Organization (WHO), cancer was the leading cause of death worldwide in 2018. Two million new cases and over 600,000 deaths were attributed to breast cancer (BC) alone [1]. In BC patients, anticancer therapy most commonly involves chemotherapy and is considered crucial in improving survival [2]. Despite its positive clinical effect, chemotherapy has been associated with debilitating side effects [3], such as muscle atrophy [4,5], cancer-related fatigue [6-8], and cardiotoxicity [9-11]. Further, a 31\% impairment in cardiorespiratory fitness (CRF), 
as measured by the peak/maximal oxygen consumption $\left(\mathrm{VO}_{2 \mathrm{max}}\right)$, has been observed in women with $\mathrm{BC}$ undergoing adjuvant chemotherapy [12]. This is a concern, given that emerging evidence suggests that CRF is a significant prognostic marker, with data indicating that a poor $\mathrm{VO}_{2 \max }$ is associated with a poorer quality of life, treatment-induced cardiotoxicity, and an increased risk of cancer-related mortality [13-16].

Aerobic training has been shown to improve CRF and other cancer-related health outcomes in cancer patients $[17,18]$, which has led major health organizations to include aerobic exercise in their physical activity guidelines for cancer patients $[19,20]$. However, the efficacy of aerobic training on CRF specifically in women with BC undergoing chemotherapy has not yet been systematically assessed, and it remains unclear what the most effective training protocols are with regard to training intensity and mode. For instance, Segal et al. (2001) [21] compared a continuous aerobic exercise guideline-based training group (chemotherapy +150 min of moderate (50-60\% of $\mathrm{VO}_{2 \max }$ ) continuously walking) versus an usual care (UC) non-exercising group. The authors showed that, after 26 weeks of training, the $\mathrm{VO}_{2 \mathrm{max}}$ decreased by $0.3 \mathrm{~mL} \cdot \mathrm{kg}^{-1} \mathrm{~min}^{-1}$ in the training group and increased by $0.2 \mathrm{~mL} \cdot \mathrm{kg}^{-1} \mathrm{~min}^{-1}$ in the UC group. Conversely, Jones et al. [22] showed that 12 weeks of moderate/vigorous-intensity aerobic training (55-100\%- $\mathrm{VO}_{2 \max }, 20-45 \mathrm{~min}-, 3$ times/week) increased the $\mathrm{VO}_{2 \max }$ (from $19.5 \pm 7.6$ to $22.1 \pm 7.0 \mathrm{~mL} \cdot \mathrm{kg}^{-1} \mathrm{~min}^{-1}$ ) in women with BC undergoing chemotherapy. Taken together, these results suggest that aerobic training intensity may play a role when aiming to improve $\mathrm{VO}_{2 m a x}$ in women with BC undergoing chemotherapy. Considering aerobic training mode, both continuous and interval aerobic training have shown to successfully impact cardiac function and hence CRF in BC patients undergoing chemotherapy [22-24]. Thus, identifying the most effective aerobic training protocols to increase $\mathrm{VO}_{2 \max }$, a strong predictor of symptom burden; cardiac function; [12] and mortality, may help to mitigate short- and long-term health issues in BC survivors.

In order to account for differences in aerobic training protocols between studies and factors such as low statistical power (small sample sizes) in randomized controlled trials, we conducted a meta-analysis. A meta-analysis summarizes data from different studies and allows testing for the effects of moderator variables (e.g., exercise intensity, mode of aerobic training), thereby improving the estimated precision of the factors affecting the changes in $\mathrm{VO}_{2 m a x}$. The purpose of this systematic review and meta-analysis was to determine: (a) the efficacy of aerobic training to increase CRF (measured by $\mathrm{VO}_{2 \max }$ ); (b) the role of aerobic training intensity (moderate or vigorous) on the $\mathrm{VO}_{2}$ max response; (c) the effect of the aerobic training mode (continuous or interval) on the changes in $\mathrm{VO}_{2 \mathrm{max}}$ in women with $\mathrm{BC}$ receiving chemotherapy.

\section{Methods}

\subsection{Protocol and Registration}

The systematic review was registered with PROSPERO (CRD42019134584), and the Preferred Reporting Items for Systematic Reviews and Meta-Analyses guidelines for systematic review reporting were followed [25].

\subsection{Search Strategy and Information Sources}

A systematic literature review was performed using three major databases (PubMed, Scopus, and Web of Science). The last search was performed on November 1st, 2019. This search was applied with no limits to publication year, type, and status. The MeSH terms were combined as follows: ((( “breast carcinoma" [Title/Abstract] OR "breast neoplasm” [Title/Abstract] OR "breast tumor" [Title/Abstract] OR "breast cancer" [Title/Abstract] OR "mammary cancer" [Title/Abstract] OR "mammary carcinoma" [Title/Abstract]))) AND ((chemotherapy [Title/Abstract] OR "adjuvant chemotherapy" [Title/Abstract] OR "neoadjuvant chemotherapy" [Title/Abstract] OR chemoradiotherapy [Title/Abstract] OR radiochemotherapy [Title/Abstract] OR "neoadjuvant therapy" [Title/Abstract] OR "adjuvant therapy" [Title/Abstract]))) AND (("exercise training" [Title/Abstract] OR 
"aerobic exercise" [Title/Abstract] OR "training exercise" [Title/Abstract] OR exercise [Title/Abstract] OR "physical exercise" [Title/Abstract] OR "endurance exercise" [Title/Abstract] OR endurance [Title/Abstract] OR "high-intensity interval training*" [Title/Abstract] OR HIIT [Title/Abstract] OR "sprint interval training*" [Title/Abstract] OR "physical activity" [Title/Abstract])). Two researchers conducted the review independently (GM and HB). Discrepancies between researchers were discussed and, if necessary, a third researcher was consulted (MSC). Reference lists of identified articles were also searched for additional relevant articles on the topic.

\subsection{Eligibility Criteria}

Only English-language studies, Pilot Studies (PS), and Randomized Controlled Trials (RCT) comparing the effects of aerobic training (continuous or interval; home-based or under professional supervision) and usual care on the $\mathrm{VO}_{2 \max }$ of women with $\mathrm{BC}$ undergoing chemotherapy (adjuvant or neoadjuvant) were included. Studies that included participants of any age with histologically confirmed BC undergoing chemotherapy in combination with other treatments were considered (e.g., radiotherapy or hormonal therapy). Studies that evaluated different types of cancer (e.g., breast, ovarian, rectal, etc.) were only included if data from a breast cancer-only group were provided. The primary outcome for this systematic review and meta-analysis was CRF, measured by the maximum oxygen uptake $\left(\mathrm{VO}_{2 \max }\right.$ or $\left.\mathrm{VO}_{2 \text { peak }}\right)$. Training intensity and mode were used as moderator variables.

The exclusion criteria were as follows: (i) duplicated articles; (ii) duplicated data; (iii) articles without original data (e.g., comments, reviews, case reports, and technical reports); (iv) studies with dietary counseling or intervention; (v) studies involving metastatic breast cancer patients; and (vi) studies with no usual care-only group.

\subsection{Study Selection and Data Extraction}

To reduce the selection bias potential, the titles and abstracts of all studies were independently evaluated by two investigators (GM and HIB). Duplicated studies were excluded, and the remaining ones were screened, assessed for eligibility criteria, and then forwarded to data extraction. Data extraction was performed by three independent reviewers (GM, HIB, and MSC) for the following variables: authors, year of publication, sample size, treatment protocol, exercise protocol, and pre and post-intervention mean \pm standard deviation (SD) values of the $\mathrm{VO}_{2 \max } / \mathrm{VO}_{2 \text { peak }}$. The data extraction procedures were standardized using a pre-piloted Excel spreadsheet. To test for possible coding drift, we randomly selected $30 \%$ of the studies for recoding following procedures outlined by Cooper et al. [26]. The mean agreement between coders was $95 \%$.

\subsection{Assessment of Risk of Bias within and across Studies and Quality}

The risk of bias of the included studies was independently assessed by two authors (CU and MSC) using the Revised Cochrane risk-of-bias tool for Randomized Trials (RoB2) [27]. The following five domains were assessed: $\{1\}$ bias arising in the randomization process; $\{2\}$ bias due to deviations from intended interventions; $\{3\}$ bias due to missing outcome data; $\{4\}$ bias in the measurement of the outcome and $\{5\}$ bias in the selection of the reported result. Each domain was classified as having $\{1\}$ LOW risk of bias; $\{2\}$ SOME CONCERN of risk of bias and \{3\} HIGH risk of bias. The Revised Cochrane risk-of-bias data is presented in Figure 1. 


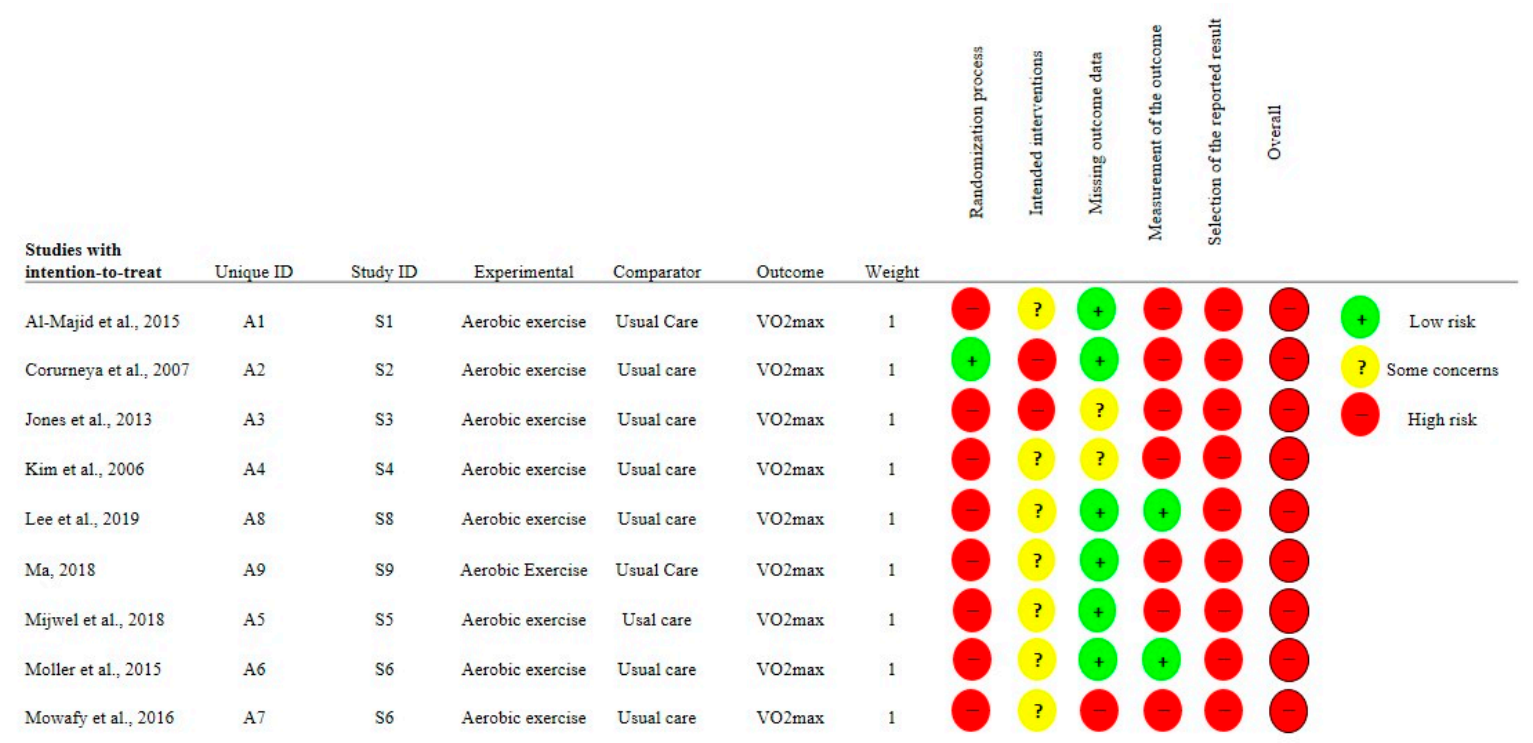

Figure 1. Risk of bias assessment for the studies included in the meta-analysis. Footnotes: $\mathrm{AE}=$ Aerobic Training; UC = Usual Care.

\subsection{Training Protocol Classification}

Each study was reviewed regarding the prescribed aerobic training protocol. Some studies implemented a periodized aerobic training protocol (i.e., progressive increase in training load), while others used a constant relative training intensity throughout the experimental period. To determine the predominant training intensity, we identified the intensity in which patients performed most of the training sessions. Training intensities were classified as moderate or vigorous. We used the American College of Sports Medicine (ACSM) equivalence table to determine the relative training intensity [28] of all the studies (Table 1). Further, the training protocols were classified as either interval or continuous training, and the total minutes of training were determined. Warm-up and cool down minutes were not included when computing the total training minutes.

It is important to highlight that seven out of nine studies measured the $\mathrm{VO}_{2 \max }$ directly. Mijwel et al. 2018 [29] estimated the $\mathrm{VO}_{2 \max }$ using a submaximal cycle ergometer test. Ma 2018 [23] indirectly measured $\mathrm{VO}_{2 \max }$ using a treadmill exhaustion protocol. Seven studies measured $\mathrm{VO}_{2}$ directly using an exhaustion protocol on a cycle ergometer (Jones et al. 2013 [22]; Lee et al. 2019 [24]; Moller et al. 2015 [30]; Mowafy et al. 2016 [31]) or treadmill (Al-Majid et al. 2015 [32]; Courneya et al. 2007 [33]; Kim et al. 2006 [34]). It is also necessary to report that five studies conducted the $\mathrm{VO}_{2 \max }$ measurement and the training on the same device [22-24,31,32], while four other studies measured the $\mathrm{VO}_{2 \max }$ and conducted the training on different devices $[29,30,33,34]$. 
Table 1. Descriptive data of the studies included in the meta-analysis.

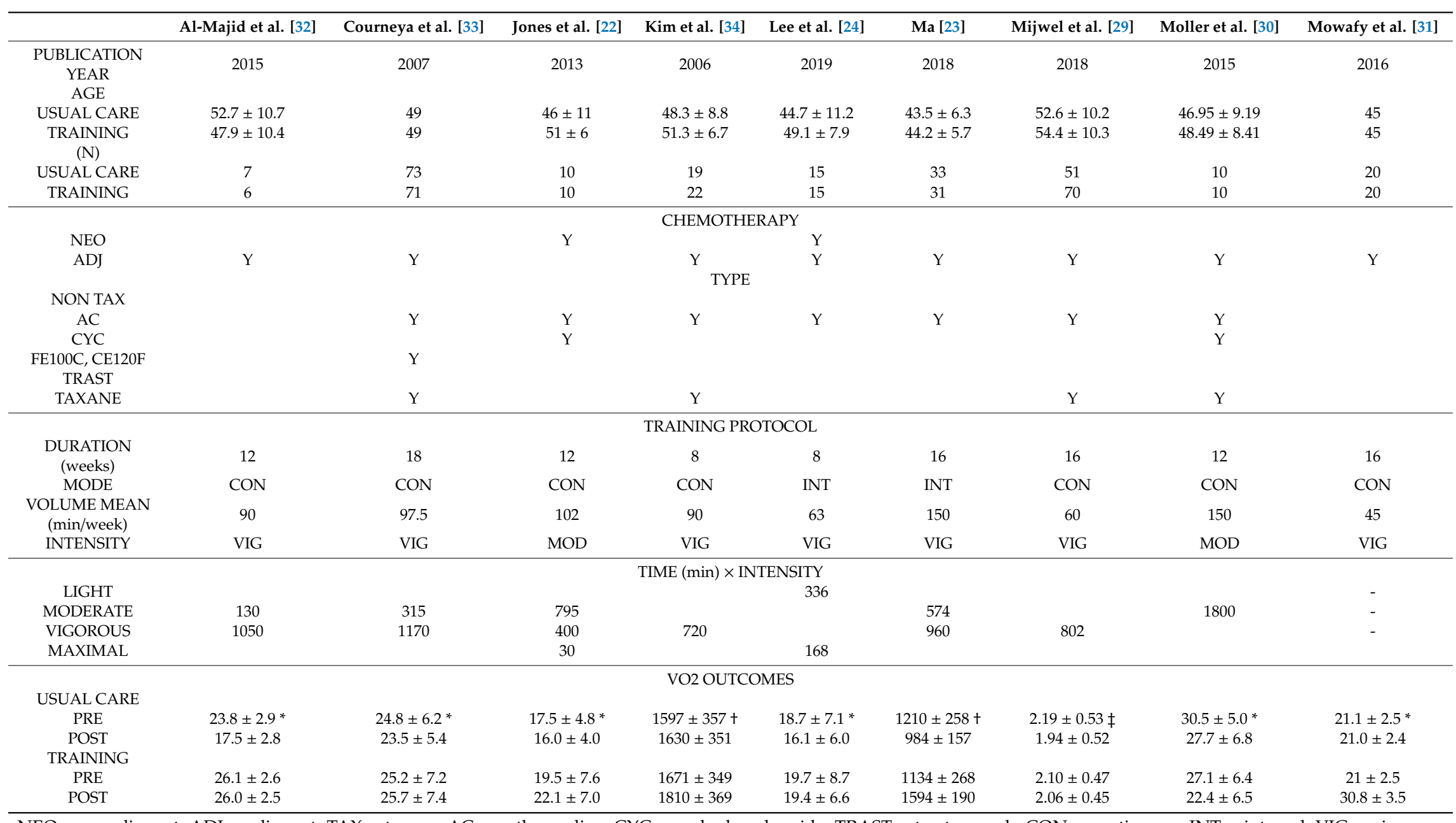

$\mathrm{NEO}=$ neoadjuvant; $\mathrm{ADJ}=$ adjuvant; $\mathrm{TAX}=$ taxane; $\mathrm{AC}=$ anthracycline $\mathrm{CYC}=$ cyclophosphamide; $\mathrm{TRAST}=$ trastuzumab; $\mathrm{CON}=$ continuous; INT $=$ interval; $\mathrm{VIG}=$ vigorous; $\mathrm{MOD}=$ moderate. $\mathrm{LIGHT}=37-45 \%$ of $\mathrm{VO}_{2 \max } ; \mathrm{MODERATE}=46-63 \%$ of $\mathrm{VO}_{2 \max } ; \mathrm{VIGOROUS}=64-90 \%$ of $\mathrm{VO}_{2 \max } ; \mathrm{MAXIMAL}=\geq 91 \%$ of $\mathrm{VO} 2 \mathrm{max} \cdot{ }^{*} \mathrm{Unit}_{\mathrm{mL}} \cdot \mathrm{kg}^{-1} \cdot \mathrm{min}{ }^{-1} ;+\mathrm{mL} \cdot \mathrm{min}{ }^{-1}$;

$\ddagger \mathrm{L} \cdot \mathrm{min}^{-1}$. It was not possible to identify the time that was performed at each training intensity; however, as the training was performed until exhaustion, it was classified as vigorous. 


\section{Statistical Analysis}

Initially, pre- to post-training effect sizes $(E S)$ were calculated according to Equation (1). Then, the between-group difference effect sizes $(d)$ for the dependent variable (i.e., maximum oxygen uptake $\left(\mathrm{VO}_{2 \max }\right)$ ) were calculated according to Equations (2-5) for each study. We estimated the pre- to post-correlation for $\mathrm{VO}_{2 \max }$ and its respective confidence interval using a bootstrapping approach based on previously published [35] and unpublished data from our group. In order to be conservative, we used the lower limit of the confidence interval obtained from the bootstrapping estimate of the pre- to post-training correlation for all studies $(r=0.611)$. The heterogeneity for between-study variability was verified with the $I^{2}$ statistics, with thresholds set as $I^{2}=25 \%$ (low), $I^{2}=50 \%$ (moderate), and $I^{2}=75 \%$ (high) [36]. Due to the high between-study heterogeneity, the data were analyzed using a random-effect model.

To investigate the potential effect of exercise intensity on the $\mathrm{VO}_{2 \max }$, we converted the exercise intensity into a categorical variable with two levels-I) low to moderate intensity; and II) vigorous intensity - which was included as a moderator variable. Similarly, the training mode was included as a categorical variable with two levels: I) continuous and II) interval training. The total volume (i.e., minutes per week) and training intervention period (i.e., the total number of training weeks) were included as continuous moderator variables in the meta-regression models. A sensitivity analysis, removing one study at a time and re-analyzing the summary effect, was performed to identify possible highly influential studies. Studies were considered influential if the removal significantly changed the summary effect (i.e., change going from significant to non-significant) [37]. Publication bias was verified using the funnel plot, Kendall's tau with continuity, and Egger's regression approaches. In cases of significant publication bias, the fill and trim procedure was implemented. All the data were analyzed using the $r m a$ and forest functions available in the metafor package for Rstudio (Version 3.6.3). The significance level was set at $p<0.05$. Data are presented as the mean \pm standard error or standard deviation and confidence interval.

$$
\begin{aligned}
& E S=\frac{\text { Mean }_{\text {post }}-\text { Mean }_{\text {pre }}}{S D_{\text {pre }}}, \\
& d=C\left[\frac{\left(\text { Mean }_{\text {posttreatment }}-\text { Mean }_{\text {pretreatment }}\right)-\left(\text { Mean }_{\text {postcontrol }}-\text { Mean }_{\text {precontrol }}\right)}{S D_{\text {changepooled }}}\right], \\
& S D_{\text {changepooled }}=\sqrt{\frac{\left(n_{\text {treatment }}{ }^{-1}\right) S D_{\text {changetreatment }}^{2}+\left(n_{\text {control }^{-1}}\right) S D_{\text {changecontrol }}^{2}}{n_{\text {treatment }}+n_{\text {control }^{-2}}}}, \\
& C=1-\frac{3}{4\left(n_{\text {treatment }}+n_{\text {control }^{-2}}\right)}, \\
& S D_{\text {change }}=\sqrt{S D_{\text {pre }}^{2}+S D_{\text {post }}^{2}-2 \cdot r_{\text {pre-postcorrelation }} \cdot S D_{\text {pre }} \cdot S D_{\text {post }}} \text {. }
\end{aligned}
$$

\section{Results}

The initial search returned 1967 studies, and 998 duplicated studies were removed. For the remaining studies (969), the titles and abstracts were screened and 919 were excluded. The remaining 50 studies were assessed for eligibility, considering our inclusion criteria. Forty-one studies were excluded, and nine studies were included in the systematic review and meta-analysis. The search and study selection process is depicted in Figure 2. 


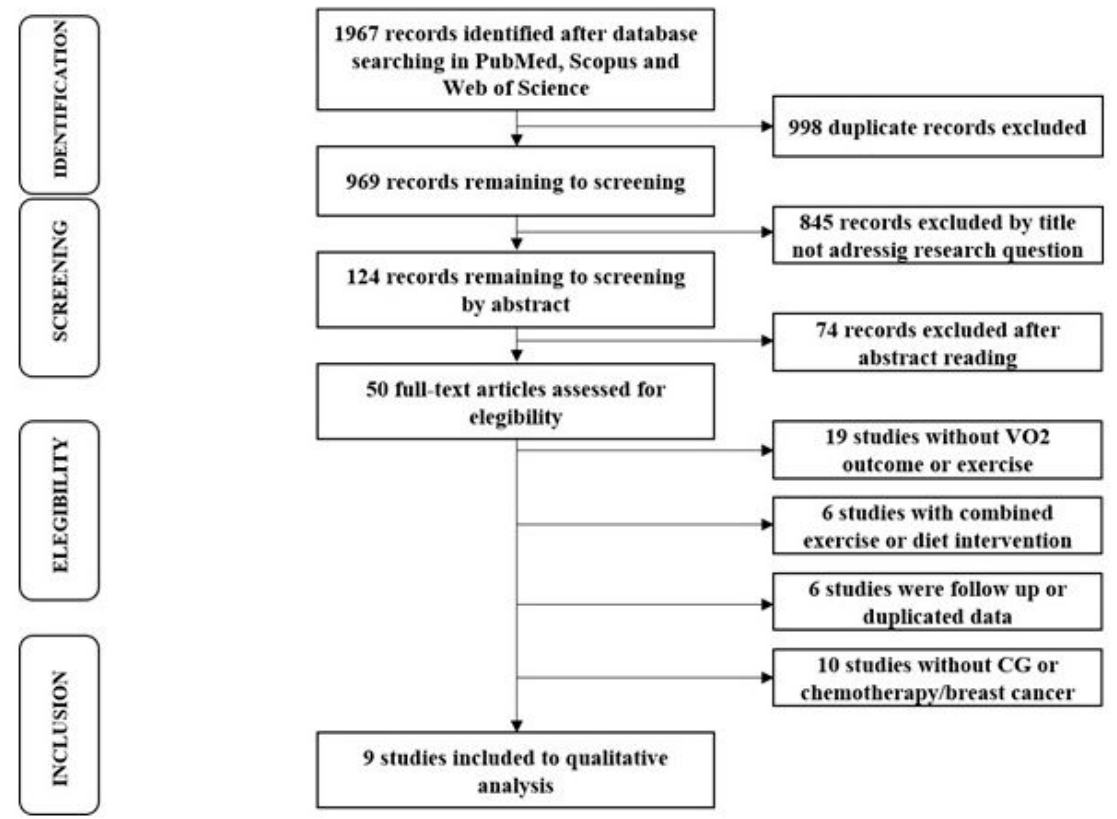

Figure 2. Flow chart of the search process.

The present meta-analysis included nine studies that compared structured aerobic training vs. usual care (i.e., no exercise), which resulted in nine treatment outcome measures. The overall number of participants in the nine studies were 493. The average pre- to post-intervention change in the $\mathrm{VO}_{2 \max }$ was $9.97 \%$ (ES: 0.62 95\% CI: -0.29 to 1.53 ) and $-10.18 \%$ (ES: $-0.54{ }_{95 \%} \mathrm{CI}:-0.99$ to -0.10 ) for the training and usual care groups, respectively. The overall between-group effect size difference for the $\mathrm{VO}_{2 \max }$ favored the aerobic training group ( $d: 1.19 \pm 0.38{ }_{95 \%} \mathrm{CI}$ : 0.45 to 1.94) (Figure 3).

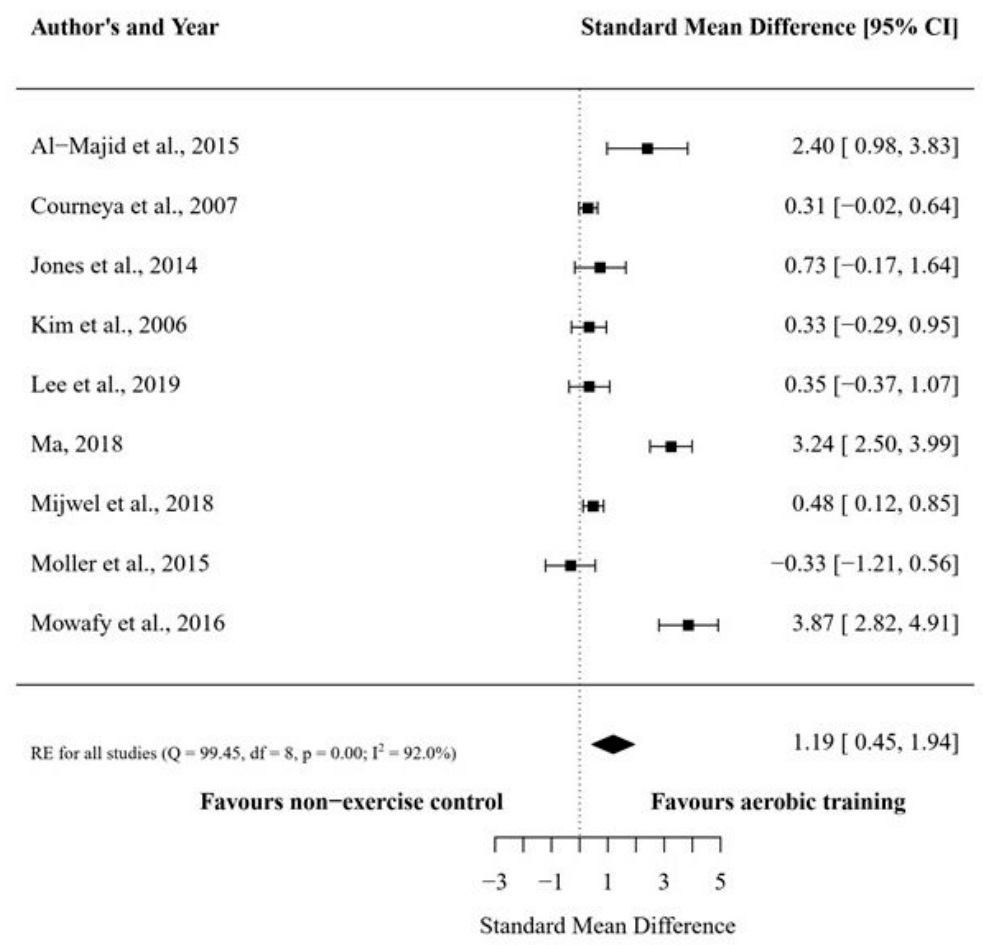

Figure 3. Forest plot for maximum oxygen consumption (VO2max) between the structured aerobic training groups and usual care (no exercise) groups. Footnotes: data are shown as between-group effect size difference $(\mathrm{d})$ and $95 \%$ confidence interval. 
The subgroup analyses revealed a significant effect favoring vigorous-intensity aerobic training protocols in increasing $\mathrm{VO}_{2 \max }$ (Table 2). Regarding the training mode/type, both continuous and interval aerobic training significantly increased the $\mathrm{VO}_{2 \max }$.

Table 2. Effects of training intensity and training mode on the maximum oxygen consumption $\left(\mathrm{VO}_{2 \max }\right)$ compared to usual care (no exercise) subgroup analyses.

\begin{tabular}{cccc}
\hline Subgroup & $\boldsymbol{N}^{\circ}$ participants & $\boldsymbol{d}(\mathbf{9 5 \%} \mathbf{C I})$ & $p$ Value \\
\hline Training intensity & & & \\
Low- to moderate [22,30] & 20 & $0.20(-1.44$ to 1.85$)$ & 0.81 \\
Vigorous [23,24,29,31-34] & 235 & $1.47(0.60$ to 2.34$)$ & 0.0009 \\
Training mode & & & \\
Continuous [22,29-34] & 209 & $1.01(0.19$ to 1.83$)$ & 0.0157 \\
Interval [23,24] & 46 & $1.79(0.28$ to 3.29$)$ & 0.02 \\
\hline
\end{tabular}

lower to moderate: $40-59 \%$ of heart rate reserve; vigorous: $60-89 \%$ of heart rate reserve; $d$ : between-group effect size difference; $I^{2}$ : heterogeneity for between-studies variability.

The meta-regression analyses did not produce significant betas for both continuous variables: total session exercise volume and total intervention period $(p>0.05)$. The sensitivity analysis demonstrated that CRF was not highly affected by any of the individual studies. Visual inspection of the funnel plot showed seven studies outside the funnel limits (five in the left and two in the right); both Kendall's tau with continuity correction (tau $=0.55 ; p=0.04$ ) and the Egger's regression intercept did not show significant bias $(z=2.0019 ; p=0.04)$ (Figure 4$)$. As the fill and trim procedure did not change the effect size estimate, we maintained the initial analysis.

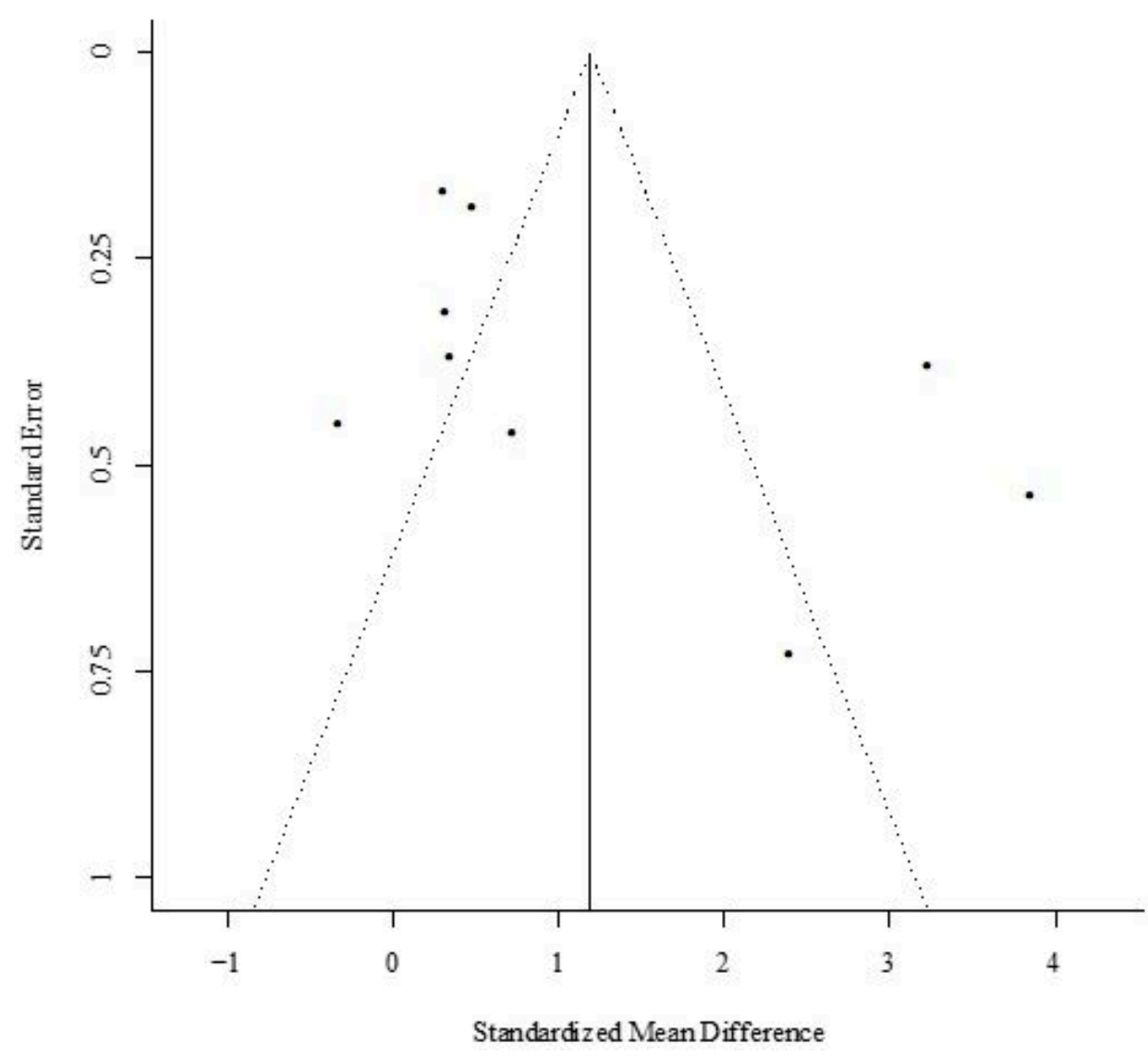

Figure 4. Funnel plot of studies comparing the maximum oxygen consumption (VO2max) between the structure aerobic training and usual care (no exercise) groups. 


\section{Discussion}

Emerging evidence on the prognostic significance of $\mathrm{CRF}$, measured by $\mathrm{VO}_{2 \max }$, underpins the clinical importance of developing effective strategies to prevent and/or recover low $\mathrm{VO}_{2 \max }$ in women with BC. Given that aerobic training is the upmost recommended exercise intervention to improve CRF, we conducted the first meta-analysis to assess: (a) the efficacy of aerobic training to increase $\mathrm{VO}_{2 \max }$; (b) the effect of moderate and vigorous intensity aerobic training on the $\mathrm{VO}_{2 \max }$ response; (c) the effect of the aerobic training mode (continuous or interval) on the changes in $\mathrm{VO}_{2 \max }$ in women with $B C$ receiving chemotherapy. Our main results show that: (a) aerobic exercise training significantly increases the $\mathrm{VO}_{2 \max }$ compared with UC; (b) only vigorous-intensity aerobic exercise $(64-90 \%$ of $\mathrm{VO}_{2 \max }$ ) significantly increases $\mathrm{VO}_{2 \max }$, with no effect for moderate-intensity aerobic protocols (46-63\% of $\mathrm{VO}_{2 \max }$ ); and (c) both continuous and interval aerobic training are effective at increasing $\mathrm{VO}_{2 \max }$. Taken together, our results suggest that, where appropriate in clinical practice, vigorous aerobic training performed with continuous or interval training mode should be considered to improve the CRF in women with BC undergoing chemotherapy.

Previous studies $[17,18]$ have demonstrated the feasibility, safety, and overall effectiveness of aerobic training for patients with BC undergoing chemotherapy; however, there is no consensus regarding the most appropriate training intensity and mode to optimize training-induced adaptations. Most studies involving BC patients have prescribed aerobic training based on guidelines from the Clinical Society of Oncology of Australia (COSA) [38], the American College of Sport Medicine (ACSM) [39], and the American Cancer Society (ACS) [40]. These guidelines recommend that patients with BC should perform 150 min of moderate or 75 min of vigorous intensity aerobic exercise per week. Our data support this recommendation, and three out of seven studies [23,31,32] (Figure 3) demonstrated that vigorous intensity aerobic training was effective in significantly improving the $\mathrm{VO}_{2 \max }$ compared to usual care. These findings are supported by a study that directly compared different exercise intensities in patients undergoing chemotherapy and found that moderate to vigorous intensity exercise led to more significant improvements in cardiorespiratory fitness and physical function and decreased the severity of adverse effects such as nausea, vomiting, pain, and physical fatigue to a greater degree than low-intensity exercise [41]. Altogether, these results suggest that, where appropriate, the prescription of vigorous intensity aerobic training should be considered for women with BC; however, additional randomized clinical trials are still necessary to substantiate both the safety and efficacy of these training protocols in larger cohorts.

Eight out of nine studies included in our review treated women with BC using anthracycline (AC)-based chemotherapy, which commonly induces cardiotoxicity, one of the most debilitating chemotherapy-related side effects [42-44]. To date, AC-induced cardiotoxicity most commonly presents as a decrease in the left ventricular ejection fraction (LVEF) [45] and, ultimately, heart failure [9,43]. Cardiotoxicity can occur at any time during AC infusion and up to years or decades later, known as late onset chronic cardiotoxicity [46,47]. A meta-analysis by Haykowsky et al. [48] showed that vigorous-to-maximal aerobic exercise was more effective than moderate-intensity exercise at improving the LVEF and $\mathrm{VO}_{2 \max }$ in patients with heart failure. A corollary from Haykowsky's study is that women with $\mathrm{BC}$ should be encouraged to perform vigorous aerobic training to prevent/treat chemotherapy-associated decreases in LVEF. Accordingly, it has been shown that vigorous exercise can prevent toxicity-related reductions in chemotherapy dose, which is critical in order to restrain tumor growth $[17,41]$. Importantly, exercise protocols with vigorous intensities have shown to produce robust increases in $\mathrm{VO}_{2 \max }$ when compared to moderate intensity protocols, regardless of the equalization in training volume [49]. Additionally, the exercise intensity seems not to affect training adherence [50]. Accordingly, higher levels of physical fitness are associated with greater adherence [51,52] and inversely associated with fatigue levels in women with BC [53].

Vigorous or high-intensity aerobic training is normally performed as interval training [54]. It is well known that the longer an individual can exercise at intensities close to the minimum velocity of $\mathrm{VO}_{2 \max }$, the greater the gains in $\mathrm{VO}_{2 \max }$ appear to be [54-56]. Due to the nature of interval 
training, which includes short sets of vigorous to maximal exercise ( $\geq 90 \%$ of $\left.\mathrm{VO}_{2 \max }\right)$ interspaced by low-intensity recovery periods, the maintenance of training intensity during aerobic exercise is possible. Our moderator analysis suggests that both continuous and interval aerobic training are effective at increasing $\mathrm{VO}_{2 \max }$. There is some compelling evidence that vigorous intensity continuous aerobic training performed during chemotherapy counteracts cancer-related fatigue, which can last up to 12 months after treatment completion, and reduces the time to return to work as compared with the UC group [57]. However, we acknowledge that performing continuous aerobic training at vigorous intensities is very demanding and may not be feasible for women with BC undergoing chemotherapy, thus interval training should be considered as a viable alternative. Additionally, some studies have demonstrated that interval training is more effective than continuous training to at increasing $\mathrm{VO}_{2 \max }$ in heart failure patients with reduced ejection fraction [58] or coronary artery disease [59], which may be an important consideration for patients with chemotherapy-associated cardiotoxicity.

The present meta-analysis has some limitations. According to our classification, only two studies assessed moderate-intensity aerobic training, and only two studies investigated an interval training mode, which makes it hard to draw definitive conclusions. Moreover, the high risk of bias of the included trials needs to be considered (Figure 1), which seems to be mainly related to the difficulty of blinding the interventions in eight out of nine included trials. It is therefore important to point out that the bias assessment for those items does not reflect a low quality of study design, but expresses the inevitable bias introduced by the lack of blinding. Another source of bias in the analysis is the CRF assessment method. Two studies assessed $\mathrm{VO}_{2 \max }$ indirectly, thus there is a small chance of bias with regard to the prescribed training intensity and training-induced increase in $\mathrm{VO}_{2 \max }$. However, as the magnitude of the effect size in Ma 2018 [23] was large and small in Mijwel et al. 2018 [29], one may suggest that the likely bias is negligible or small. It is reasonable to suggest that the differences in effect sizes were due to the higher training volume and more intensive training regimen. Thus, larger and better blinding-controlled trials should be conducted to properly resolve this issue.

The data of the present manuscript indicate that high-intensity aerobic training can be performed by women with $B C$ under chemotherapy regardless of the training method (continuous or interval training), however there are several considerations that need to be taken into account in clinical practice. High-intensity continuous training (walking/run, 5x week at $70 \%$ of $\mathrm{VO}_{2 \text { peak }}$ for $30 \mathrm{~min}$ ) may be very demanding for this cohort. Thus, performing high-intensity interval training (e.g., cycling $3 \mathrm{x}$ of $3 \mathrm{~min}$ at $90 \%$ of $\mathrm{VO}_{2 \text { peak, }}$, with intervals cycling at $30 \%$ of $\mathrm{VO}_{2 \text { peak }}, 3 x$ week) seems to be a more feasible alternative, as it is less time-consuming and more enjoyable. Further, while several studies prescribe exercise intensity based on heart rate, chemotherapy may influence a patient's heart rate. Although heart rate can be easily monitored on a daily basis, and heart hate reserve can be calculated, the training intensity can be influenced. Hence, a watt-based training prescription represents a less error-prone method. On the other hand, there is mounting evidence that breast cancer chemotherapy protocols elicit high levels of fatigue, which can greatly decrease a patient's ability to maintain a target Wattage. Finally, when the training sessions are not performed with the same equipment (e.g., stationary bike, treadmill) that is used for the CRF assessment, the training intensity may have been a little under due to non-specific training-induced peripheral adaptations (i.e., at the skeletal muscle level).

\section{Conclusions}

In summary, our findings indicated that aerobic exercise increases the $\mathrm{VO}_{2 \max }$ in women with $\mathrm{BC}$ undergoing chemotherapy. We also showed that vigorous intensities $\left(64-90 \%\right.$ of $\left.\mathrm{VO}_{2 \max }\right)$ performed with continuous or interval aerobic training are effective at increasing the $\mathrm{VO}_{2 \max }$ in women with $\mathrm{BC}$ undergoing chemotherapy. Performing continuous aerobic training at vigorous intensities is very demanding and, thus, interval aerobic training should be considered as a viable option for women with BC. While this work supports the benefits of aerobic exercise, additional clinical investigations are warranted to determine the effects of different exercise modalities, timings, and durations and to identify 
optimal aerobic training regimens to not only improve CRF but also counteract treatment-related side-effects, such as cardiotoxicity, in women with BC.

Author Contributions: Conceptualization: G.M., E.Z., M.S.C.; Performed searches: G.M., H.I.B., and M.S.C.; Data extraction: G.M., H.I.B., G.D.T., M.S.C.; Performed risk of bias assessment: C.U., F.C.V., M.S.C.; Methodology: F.C.V., M.E.L., C.U.; Software: M.E.L., C.U., L.O.S.; Supervision: S.D., M.S.C.; Writing-original draft: G.M., M.E.L., C.U., S.D., M.S.C. All the authors have read and agreed to the published version of the manuscript. Please turn to the CRediT taxonomy for the term explanation. Authorship must be limited to those who have contributed substantially to the work reported. All authors have read and agreed to the published version of the manuscript.

Funding: No external sources of funding were used in the preparation of this manuscript.

Acknowledgments: The authors would like to express gratitude to the São Paulo Research Foundation (FAPESP; Grant No. 2015/19756-3), the National Council for Scientific and Technological Development (CNPq grant No. 303742/2018-6 and No. 303085/2015-0), and the Coordination of Improvement of Higher Education Personnel-Brazil (CAPES grant \#001).

Conflicts of Interest: The authors declare no conflict of interest.

\section{References}

1. World Health Organization International Agency for Research on Cancer. Globocan. 2018. Available online: http://gco.iarc.fr/today (accessed on 12 November 2019).

2. Jemal, A.; Ward, E.M.; Johnson, C.J.; Cronin, K.A.; Ma, J.; Ryerson, A.B.; Mariotto, A.; Lake, A.J.; Wilson, R.; Sherman, R.L.; et al. Annual Report to the Nation on the Status of Cancer, 1975-2014, Featuring Survival. JNCI J. Natl. Cancer Inst. 2017, 109, 1-22. [CrossRef]

3. Partridge, A.H.; Burstein, H.J.; Winer, E.P. Side Effects of Chemotherapy and Combined Chemohormonal Therapy in Women with Early-Stage Breast Cancer. JNCI Monogr. 2001, 2001, 135-142. [CrossRef] [PubMed]

4. Mazzuca, F.; Onesti, C.E.; Roberto, M.; Di Girolamo, M.; Botticelli, A.; Begini, P.; Strigari, L.; Marchetti, P.; Muscaritoli, M. Lean body mass wasting and toxicity in early breast cancer patients receiving anthracyclines. Oncotarget 2018, 9, 25714-25722. [CrossRef] [PubMed]

5. Freedman, R.J.; Aziz, N.; Albanes, D.; Hartman, T.; Danforth, D.; Hill, S.; Sebring, N.; Reynolds, J.C.; Yanovski, J.A. Weight and Body Composition Changes during and after Adjuvant Chemotherapy in Women with Breast Cancer. J. Clin. Endocrinol. Metab. 2004, 89, 2248-2253. [CrossRef] [PubMed]

6. Tavio, M.; Milan, I.; Tirelli, U. Cancer-related fatigue (review). Int. J. Oncol. 2002, 21, 1093-1099. [CrossRef]

7. Campos, M.P.O.; Hassan, B.J.; Riechelmann, R.; Del Giglio, A. Cancer-related fatigue: A practical review. Ann. Oncol. 2011, 22, 1273-1279. [CrossRef]

8. Hofman, M.; Ryan, J.L.; Figueroa-Moseley, C.D.; Jean-Pierre, P.; Morrow, G.R. Cancer-related fatigue: The scale of the problem. Oncologist 2007, 12, 4-10. [CrossRef]

9. Von Hoff, D.D.; Layard, M.W.; Basa, P.; Davis, H.L., Jr.; Von Hoff, A.L.; Rozencweig, M.; Muggia, F.M. Risk Factors for Doxorubicin-lnduced Congestive Heart Failure. Ann. Intern. Med. 1979, 91, 710. [CrossRef]

10. Khouri, M.G.; Douglas, P.S.; Mackey, J.R.; Martin, M.; Scott, J.M.; Scherrer-Crosbie, M.; Jones, L.W. Cancer therapy-induced cardiac toxicity in early breast cancer: Addressing the unresolved issues. Circulation 2012, 126, 2749-2763. [CrossRef]

11. Yeh, E.T.H.; Bickford, C.L. Cardiovascular complications of cancer therapy: Incidence, pathogenesis, diagnosis, and management. J. Am. Coll. Cardiol. 2009, 53, 2231-2247. [CrossRef]

12. Jones, L.W.; Courneya, K.S.; Mackey, J.R.; Muss, H.B.; Pituskin, E.N.; Scott, J.M.; Hornsby, W.E.; Coan, A.D.; Herndon, J.E.; Douglas, P.S.; et al. Cardiopulmonary Function and Age-Related Decline Across the Breast Cancer Survivorship Continuum. J. Clin. Oncol. 2012, 30, 2530-2537. [CrossRef] [PubMed]

13. Sloan, R.A.; Sawada, S.S.; Martin, C.K.; Church, T.; Blair, S.N. Associations between cardiorespiratory fitness and health-related quality of life. Health Qual. Life Outcomes 2009, 7, 3-7. [CrossRef] [PubMed]

14. Schmid, D.; Leitzmann, M.F. Cardiorespiratory fitness as predictor of cancer mortality: A systematic review and meta-analysis. Ann. Oncol. 2015, 26, 272-278. [CrossRef]

15. Herrero, F.; Balmer, J.; San Juan, A.F.; Foster, C.; Fleck, S.J.; Perez, M.; Canete, S.; Earnest, C.P.; Lucia, A. Is cardiorespiratory fitness related to quality of life in survivors of breast cancer? J. Strength Cond. Res. 2006, 20, 535-540. [CrossRef] 
16. Yu, A.F.; Flynn, J.R.; Moskowitz, C.S.; Scott, J.M.; Oeffinger, K.C.; Dang, C.T.; Liu, J.E.; Jones, L.W.; Steingart, R.M. Long-term Cardiopulmonary Consequences of Treatment-Induced Cardiotoxicity in Survivors of ERBB2 -Positive Breast Cancer. JAMA Cardiol. 2020, 5, 309. [CrossRef] [PubMed]

17. Furmaniak, A.C.; Menig, M.; Markes, M.H. Exercise for women receiving adjuvant therapy for breast cancer. Cochrane Database Syst. Rev. 2016, 9, CD005001. [CrossRef]

18. Singh, B.; Spence, R.R.; Steele, M.L.; Sandler, C.X.; Peake, J.M.; Hayes, S.C. A Systematic Review and Meta-Analysis of the Safety, Feasibility, and Effect of Exercise in Women with Stage II+ Breast Cancer. Arch. Phys. Med. Rehabil. 2018, 99, 2621-2636. [CrossRef]

19. Hayes, S.C.; Newton, R.U.; Spence, R.R.; Galvao, D.A. The Exercise and Sports Science Australia position statement: Exercise medicine in cancer management. J. Sci. Med. Sport 2019, 22, 1175-1199. [CrossRef]

20. Campbell, K.L.; Winters-Stone, K.M.; Wiskemann, J.; May, A.M.; Schwartz, A.L.; Courneya, K.S.; Zucker, D.S.; Matthews, C.E.; Ligibel, J.A.; Gerber, L.H.; et al. Exercise Guidelines for Cancer Survivors: Consensus Statement from International Multidisciplinary Roundtable. Med. Sci. Sports Exerc. 2019, 51, 2375-2390. [CrossRef]

21. Segal, R.; Evans, W.; Johnson, D.; Smith, J.; Colletta, S.; Gayton, J.; Woodard, S.; Wells, G.; Reid, R. Structured Exercise Improves Physical Functioning in Women with Stages I and II Breast Cancer: Results of a Randomized Controlled Trial. J. Clin. Oncol. 2001, 19, 657-665. [CrossRef]

22. Jones, L.W.; Fels, D.R.; West, M.; Allen, J.D.; Broadwater, G.; Barry, W.T.; Wilke, L.G.; Masko, E.; Douglas, P.S.; Dash, R.C.; et al. Modulation of Circulating Angiogenic Factors and Tumor Biology by Aerobic Training in Breast Cancer Patients Receiving Neoadjuvant Chemotherapy. Cancer Prev. Res. 2013, 6, 925-937. [CrossRef] [PubMed]

23. Ma, Z. Effect of anthracycline combined with aerobic exercise on the treatment of breast cancer. Pak. J. Pharm. Sci. 2018, 31, 1125-1129. [PubMed]

24. Lee, K.; Kang, I.; Mack, W.J.; Mortimer, J.; Sattler, F.; Salem, G.; Dieli-Conwright, C.M. Feasibility of high intensity interval training in patients with breast Cancer undergoing anthracycline chemotherapy: A randomized pilot trial. BMC Cancer 2019, 19, 653. [CrossRef] [PubMed]

25. Moher, D.; Liberati, A.; Tetzlaff, J.; Altman, D.G.; Grp, P. Preferred Reporting Items for Systematic Reviews and Meta-Analyses: The PRISMA Statement (Reprinted from Annals of Internal Medicine). Phys. Ther. 2009, 89, 873-880. [CrossRef]

26. Cooper, H.; Hedges, L.V.; Valentine, J.C. (Eds.) Handbook of Research Synthesis and Meta-Analysis, 3rd ed.; Russell Sage Foundation: New York, NY, USA, 2009; ISBN 9780871541635.

27. Sterne, J.A.C.; Savovic, J.; Page, M.J.; Elbers, R.G.; Blencowe, N.S.; Boutron, I.; Cates, C.J.; Cheng, H.-Y.; Corbett, M.S.; Eldridge, S.M.; et al. RoB 2: A revised tool for assessing risk of bias in randomised trials. BMJ 2019, 366, 14898. [CrossRef]

28. Garber, C.E.; Blissmer, B.; Deschenes, M.R.; Franklin, B.A.; Lamonte, M.J.; Lee, I.-M.; Nieman, D.C.; Swain, D.P. Quantity and Quality of Exercise for Developing and Maintaining Cardiorespiratory, Musculoskeletal, and Neuromotor Fitness in Apparently Healthy Adults. Med. Sci. Sport. Exerc. 2011, 43, 1334-1359. [CrossRef]

29. Mijwel, S.; Backman, M.; Bolam, K.A.; Olofsson, E.; Norrbom, J.; Bergh, J.; Sundberg, C.J.; Wengström, Y.; Rundqvist, H. Highly favorable physiological responses to concurrent resistance and high-intensity interval training during chemotherapy: The OptiTrain breast cancer trial. Breast Cancer Res. Treat. 2018, 169, 93-103. [CrossRef]

30. Møller, T.; Lillelund, C.; Andersen, C.; Bloomquist, K.; Christensen, K.B.; Ejlertsen, B.; Nørgaard, L.; Wiedenbein, L.; Oturai, P.; Breitenstein, U.; et al. The challenge of preserving cardiorespiratory fitness in physically inactive patients with colon or breast cancer during adjuvant chemotherapy: A randomised feasibility study. BMJ Open Sport Exerc. Med. 2015, 1, e000021. [CrossRef]

31. Mowafy, Z.M.E.; Zoheiry, I.M.I.; Elmonem, M.G.A.; Katter, D. Efficacy of aerobic training on maximal oxygen consumption and total leukocytes count after chemotherapy in breast cancer patients. Int. J. PharmTech Res. 2016, 9, 34-40. 
32. Al-Majid, S.; Wilson, L.D.; Rakovski, C.; Coburn, J.W. Effects of Exercise on Biobehavioral Outcomes of Fatigue During Cancer Treatment. Biol. Res. Nurs. 2015, 17, 40-48. [CrossRef]

33. Courneya, K.S.; Segal, R.J.; Mackey, J.R.; Gelmon, K.; Reid, R.D.; Friedenreich, C.M.; Ladha, A.B.; Proulx, C.; Vallance, J.K.H.; Lane, K.; et al. Effects of Aerobic and Resistance Exercise in Breast Cancer Patients Receiving Adjuvant Chemotherapy: A Multicenter Randomized Controlled Trial. J. Clin. Oncol. 2007, 25, 4396-4404. [CrossRef] [PubMed]

34. Kim, C.-J.; Kang, D.; Smith, B.A.; Landers, K.A. Cardiopulmonary responses and adherence to exercise in women newly diagnosed with breast cancer undergoing adjuvant therapy. Cancer Nurs. 2006, 29, 156-165. [CrossRef] [PubMed]

35. Conceicao, M.S.; Junior, E.M.M.; Telles, G.D.; Libardi, C.A.; Castro, A.; Andrade, A.L.L.; Brum, P.C.; Urias, U.; Kurauti, M.A.; Junior, J.M.C.; et al. Augmented Anabolic Responses after 8-wk Cycling with Blood Flow Restriction. Med. Sci. Sports Exerc. 2019, 51, 84-93. [CrossRef] [PubMed]

36. Higgins, J.P.T. Measuring inconsistency in meta-analyses. BMJ 2003, 327, 557-560. [CrossRef]

37. Lixandrão, M.E.; Ugrinowitsch, C.; Berton, R.; Vechin, F.C.; Conceição, M.S.; Damas, F.; Libardi, C.A.; Roschel, H. Magnitude of Muscle Strength and Mass Adaptations Between High-Load Resistance Training Versus Low-Load Resistance Training Associated with Blood-Flow Restriction: A Systematic Review and Meta-Analysis. Sport. Med. 2018, 48, 361-378. [CrossRef]

38. Cormie, P.; Atkinson, M.; Bucci, L.; Cust, A.; Eakin, E.; Hayes, S.; McCarthy, S.; Murnane, A.; Patchell, S.; Adams, D. Clinical Oncology Society of Australia position statement on exercise in cancer care. Med. J. Aust. 2018, 209, 184-187. [CrossRef]

39. Schmitz, K.H.; Courneya, K.S.; Matthews, C.; Demark-Wahnefried, W.; Galvão, D.A.; Pinto, B.M.; Irwin, M.L.; Wolin, K.Y.; Segal, R.J.; Lucia, A.; et al. American College of Sports Medicine Roundtable on Exercise Guidelines for Cancer Survivors. Med. Sci. Sport. Exerc. 2010, 42, 1409-1426. [CrossRef]

40. Park, S.-H.; Knobf, M.T.; Kerstetter, J.; Jeon, S. Adherence to American Cancer Society Guidelines on Nutrition and Physical Activity in Female Cancer Survivors. Cancer Nurs. 2018, 42, 1. [CrossRef]

41. van Waart, H.; Stuiver, M.M.; van Harten, W.H.; Geleijn, E.; Kieffer, J.M.; Buffart, L.M.; de Maaker-Berkhof, M.; Boven, E.; Schrama, J.; Geenen, M.M.; et al. Effect of Low-Intensity Physical Activity and Moderateto High-Intensity Physical Exercise During Adjuvant Chemotherapy on Physical Fitness, Fatigue, and Chemotherapy Completion Rates: Results of the PACES Randomized Clinical Trial. J. Clin. Oncol. 2015, 33, 1918-1927. [CrossRef]

42. Volkova, M.; Russell, R. Anthracycline cardiotoxicity: Prevalence, pathogenesis and treatment. Curr. Cardiol. Rev. 2011, 7, 214-220. [CrossRef]

43. Swain, S.M.; Whaley, F.S.; Ewer, M.S. Congestive heart failure in patients treated with doxorubicin: A retrospective analysis of three trials. Cancer 2003, 97, 2869-2879. [CrossRef] [PubMed]

44. Buzdar, A.U.; Marcus, C.; Smith, T.L.; Blumenschein, G.R. Early and delayed clinical cardiotoxicity of doxorubicin. Cancer 1985, 55, 2761-2765. [CrossRef]

45. Scott, J.M.; Khakoo, A.; Mackey, J.R.; Haykowsky, M.J.; Douglas, P.S.; Jones, L.W. Modulation of Anthracycline-Induced Cardiotoxicity by Aerobic Exercise in Breast Cancer. Circulation 2011, 124, 642-650. [CrossRef] [PubMed]

46. Mulrooney, D.A.; Yeazel, M.W.; Kawashima, T.; Mertens, A.C.; Mitby, P.; Stovall, M.; Donaldson, S.S.; Green, D.M.; Sklar, C.A.; Robison, L.L.; et al. Cardiac outcomes in a cohort of adult survivors of childhood and adolescent cancer: retrospective analysis of the Childhood Cancer Survivor Study cohort. BMJ 2009, 339, b4606. [CrossRef] [PubMed]

47. Kremer, L.C.; van Dalen, E.C.; Offringa, M.; Ottenkamp, J.; Voute, P.A. Anthracycline-induced clinical heart failure in a cohort of 607 children: Long-term follow-up study. J. Clin. Oncol. 2001, 19, 191-196. [CrossRef]

48. Haykowsky, M.J.; Timmons, M.P.; Kruger, C.; McNeely, M.; Taylor, D.A.; Clark, A.M. Meta-analysis of aerobic interval training on exercise capacity and systolic function in patients with heart failure and reduced ejection fractions. Am. J. Cardiol. 2013, 111, 1466-1469. [CrossRef]

49. Milanović, Z.; Sporiš, G.; Weston, M. Effectiveness of High-Intensity Interval Training (HIT) and Continuous Endurance Training for VO2max Improvements: A Systematic Review and Meta-Analysis of Controlled Trials. Sport. Med. 2015, 45, 1469-1481. [CrossRef] 
50. Courneya, K.S.; Segal, R.J.; Gelmon, K.; Mackey, J.R.; Friedenreich, C.M.; Yasui, Y.; Reid, R.D.; Proulx, C.; Trinh, L.; Dolan, L.B.; et al. Predictors of adherence to different types and doses of supervised exercise during breast cancer chemotherapy. Int. J. Behav. Nutr. Phys. Act. 2014, 11, 85. [CrossRef]

51. Kampshoff, C.S.; Jansen, F.; van Mechelen, W.; May, A.M.; Brug, J.; Chinapaw, M.J.M.; Buffart, L.M. Determinants of exercise adherence and maintenance among cancer survivors: A systematic review. Int. J. Behav. Nutr. Phys. Act. 2014, 11, 80. [CrossRef]

52. van Waart, H.; Buffart, L.M.; Stuiver, M.M.; van Harten, W.H.; Sonke, G.S.; Aaronson, N.K. Adherence to and satisfaction with low-intensity physical activity and supervised moderate-high intensity exercise during chemotherapy for breast cancer. Support. Care Cancer 2020, 28, 2115-2126. [CrossRef]

53. Huang, H.-P.; Wen, F.-H.; Tsai, J.-C.; Lin, Y.-C.; Shun, S.-C.; Chang, H.-K.; Wang, J.-S.; Jane, S.-W.; Chen, M.-C.; Chen, M.-L. Adherence to prescribed exercise time and intensity declines as the exercise program proceeds: Findings from women under treatment for breast cancer. Support. Care Cancer 2015, 23, 2061-2071. [CrossRef] [PubMed]

54. Buchheit, M.; Laursen, P.B. High-intensity interval training, solutions to the programming puzzle: Part I: Cardiopulmonary emphasis. Sport. Med. 2013, 43, 313-338. [CrossRef] [PubMed]

55. Buchheit, M.; Laursen, P.B. High-intensity interval training, solutions to the programming puzzle: Part II: Anaerobic energy, neuromuscular load and practical applications. Sport. Med. 2013, 43, 927-954. [CrossRef] [PubMed]

56. Midgley, A.W.; Mc Naughton, L.R. Time at or near VO2max during continuous and intermittent running. A review with special reference to considerations for the optimisation of training protocols to elicit the longest time at or near VO2max. J. Sports Med. Phys. Fit. 2006, 46, 1-14.

57. Mijwel, S.; Jervaeus, A.; Bolam, K.A.; Norrbom, J.; Bergh, J.; Rundqvist, H.; Wengström, Y. High-intensity exercise during chemotherapy induces beneficial effects 12 months into breast cancer survivorship. J. Cancer Surviv. 2019, 13, 244-256. [CrossRef]

58. Gomes Neto, M.; Durães, A.R.; Conceição, L.S.R.; Saquetto, M.B.; Ellingsen, Ø.; Carvalho, V.O. High intensity interval training versus moderate intensity continuous training on exercise capacity and quality of life in patients with heart failure with reduced ejection fraction: A systematic review and meta-analysis. Int. J. Cardiol. 2018, 261, 134-141. [CrossRef]

59. Liou, K.; Ho, S.; Fildes, J.; Ooi, S.-Y. High Intensity Interval versus Moderate Intensity Continuous Training in Patients with Coronary Artery Disease: A Meta-analysis of Physiological and Clinical Parameters. Heart Lung Circ. 2016, 25, 166-174. [CrossRef] 